\title{
Radiating Regions in Pulsar Magnetospheres: From Theory to Observations and Back
}

\author{
Vladimir V. Usov \\ Department of Condensed-Matter Physics, Weizmann Institute of \\ Science, Rehovot 76100, Israel
}

\begin{abstract}
We discuss plausible locations of radiating regions in the magnetospheres of pulsars and argue that the mechanisms of pulsar radiation at different frequencies are closely connected with the locations of the radiating regions, especially in the radio range. If the region that is responsible for the bulk of the non-thermal radiation at some frequency is localized in the pulsar magnetosphere then the nature of this radiation will be either determined or, at least, restricted significantly.
\end{abstract}

\section{Introduction}

Over 30 years since the discovery of pulsars, the mechanism of their radio emission is still poorly understood. Moreover, the location of radio emitting regions in the pulsar magnetospheres is unknown. Strong non-thermal high-frequency (optical, X-ray and $\gamma$-ray) radiation is observed from about ten pulsars. The pulses of high-frequency radiation generally bear little resemblance to the radio pulses (with the exception of the Crab pulsar). Besides, at different frequencies the pulses of the high-frequency radiation are different. These imply that there are many regions in the pulsar magnetospheres where strong non-thermal radiation is generated.

\section{Radiating Regions in Pulsar Magnetospheres}

A common point of all acceptable models of pulsars is that a strong electric field $\mathbf{E}_{\|}=(\mathbf{E} \cdot \mathbf{B}) \mathbf{B} /|\mathbf{B}|^{2}$ along the magnetic field $\mathbf{B}$ is generated in the pulsar magnetospheres. Primary particles are accelerated by such a field to ultrarelativistic energies and generate $\gamma$-rays. Some of these $\gamma$-rays are absorbed by creating secondary electron-positron pairs. The created pairs screen the electric field $\mathbf{E}_{\|}$ in the pulsar magnetosphere everywhere except for compact regions. The compact regions where $\mathbf{E}_{\|}$is unscreened are called gaps. These gaps are "engines" that are responsible for the non-thermal radiation of pulsars. Most probably, the gaps are located either near the magnetic poles of pulsars or near their light cylinders (for a review, see Michel 1991). Some part, of radiation generated in the gaps and their vicinities escapes from the pulsar magnetospheres (see below) and may be observed. Plasma instabilities may be developed in the secondary electron-positron plasma. The regions of their development are also plausible sources of the non-thermal radiation of pulsars in addition to the gaps. 


\subsection{Radiation from Polar Gaps}

Gaps that form near the magnetic poles of pulsars are called polar gaps. Physical processes (acceleration of particles, generation of radiation and its propagation, creation of electron-positron pairs, etc.) in the polar gaps and their vicinities have been discussed in many papers (e.g., Usov 1996; Zhang \& Harding 1999). The bulk of the polar gap radiation is in hard X-rays and $\gamma$-rays. In all conventional polar-gap models (Ruderman \& Sutherland 1975; Arons 1981; Zhang \& Harding 1999 and references therein) where created electron-positron pairs are free the total power carried away by both relativistic particles and radiation from the polar gap into the pulsar magnetosphere is the same within a factor of $2-3$ or so. This power is about ten times less than the $\gamma$-ray luminosity, at least for the most part of pulsars detected in $\gamma$-rays.

It was shown (Shabad \& Usov 1986) that in a strong magnetic field, $B>$ $0.1 B_{\mathrm{cr}}, \gamma$-rays emitted nearly along curved magnetic field lines adiabatically convert into bound pairs (positronium atoms) rather than decaying into free pairs, where $B_{\mathrm{cr}}=4.4 \times 10^{13} \mathrm{G}$. This partially prevents the screening of the vacuum electric field near the pulsar surface, and the total power carried away by both relativistic particles and radiation from the polar gap into the pulsar magnetosphere can increase significantly. For pulsars with a strong magnetic field at their surface, $B_{\mathrm{s}}>0.1 B_{\mathrm{cr}}$, a modified polar gap model was developed (Usov \& Melrose 1995, 1996). In this model, the non-thermal luminosity of pulsars may be comparable to the rate of rotational energy losses that is enough to explain the observed luminosities of all known $\gamma$-ray pulsars.

A maser version of linear acceleration emission was suggested as a mechanism of radio radiation from the polar gaps of pulsars (e.g., Melrose 1978; Rowe 1992). This mechanism requires an oscillating electric field $\mathbf{E}_{\|}$in the polar gaps. The characteristic frequency of the radio emission is $\sim \omega_{0} \Gamma^{2}$, where $\omega_{0}$ is the oscillation frequency, and $\Gamma$ is the Lorentz-factor of radiating particles. In this model, the radio beam coincides with the beam of high-frequency (X-ray and $\gamma$-ray) emission from the polar gaps.

\subsection{Plasma Instabilities in Pulsar Magnetospheres and Non-thermal Radiation from the Regions of their Development}

Two-stream instability. It was argued that the process of pair creation is strongly nonstationary, and the pair plasma that flows away from the pulsar surface is nonhomogeneous and gathers into separate closes (e.g., Usov 1987). Since the Lorentz-factors of the pair plasma lie within a wide range (from $\Gamma_{\min } \sim 10$ to $\left.\Gamma_{\max } \sim 10^{4}-10^{5}\right)$, the high-energy particles $\left(\Gamma \sim \Gamma_{\max }\right)$ go ahead, and the plasma clouds disperse as they go further from the pulsar. At a distance of $\sim 2 l \Gamma_{\min }^{2}$ from the pulsar surface the high-energy particles of a given cloud catch up with the low-energy particles $\left(\Gamma \sim \Gamma_{\min }\right)$ that belong to the cloud going ahead of it, where $l$ is the characteristic length between the clouds at the moment of their creation near the pulsar surface. In the cloud overlapping region the energy distribution of particles is two-humped, i.e., there are particles only with both $\Gamma \sim \Gamma_{\min }$ and $\Gamma \sim \Gamma_{\max }$ whereas particles with intermediate Lorentz-factors are absent. The plasma with such a distribution is unstable with respect to twostream instability (Usov 1987; Ursov \& Usov 1988; Asseo \& Melikidze 1998). Longitudinal (nonescaping) Langmuir waves that are generated in the process 
of development of this instability may be converted by means of different nonlinear effects into electromagnetic waves that can escape from the pulsar magnetosphere (e.g., Lesch, Gil, \& Shukla 1994; Lyubarskii 1996; Mahajan, Machabeli, \& Rogova 1997; Melikidze, Gil, \& Pataraya 2000). This non-linear conversion of waves is a "bottle-neck" that impedes the meeting of the model of pulsar radio emission and observational data. The two-stream instability of strongly nonhomogeneous plasma develops very fast, and if the process of pair creation near the pulsar surface is strongly nonstationary indeed, the development of this instability in the pulsar magnetospheres is almost inevitable. It is worth noting that the outflowing plasma may have several characteristic lengths of its modulation. For instance, in the Ruderman-Sutherland model they are $l_{1} \simeq 0.3 R$ and $l_{2} \simeq 2 R_{c}$, where $R$ is the neutron star radius and $R_{c}$ is the curvature radius of magnetic field lines at the pulsar surface. For $R_{c} \simeq R \simeq 10^{6} \mathrm{~cm}$ and $\Gamma_{\min } \simeq 10$, the distances to the radio emitting regions are $\sim 30 R$ and $\sim 200 R$. These distances are consistent with observational data (Rankin 1993; Kijak \& Gil 1997 and references therein). In this model, high-frequency radiation from the radio emitting regions is very weak.

Cyclotron instability. Near the light cylinders of pulsars, $r \sim c / \Omega$, the outflowing plasma may be unstable with respect to excitation of cyclotron waves (Machabeli \& Usov 1979), where $c$ is the speed of light, $\Omega=2 \pi / P$ and $P$ is the pulsar period. For typical pulsars, $B_{\mathrm{s}} \sim 10^{12} \mathrm{G}$ and $P \sim 1 \mathrm{~s}$, the frequency of these waves is in the radio range, from $\sim 10^{2} \mathrm{MHz}$ to a few $\times 10^{3} \mathrm{MHz}$ (Machabeli \& Usov 1989). The model that is based on the cyclotron instability also can explain many observational data on radio emission of pulsars (Machabeli \& Usov 1989; Lyutikov, Blandford, \& Machabeli 2000). The interaction between cyclotron waves and outflowing particles leads to diffusion of particles in the momentum space across the magnetic field. As a result, outflowing particles acquire non-zero pitch angles and generate high-frequency radiation via the synchrotron mechanism. The high-frequency luminosity of the region where the cyclotron instability develops may be as high as the power carried away by particles from the polar gap into the pulsar magnetosphere.

\subsection{Radiation from Outer Gaps}

Charge deficient regions (outer gaps) with a strong electric field $E_{\|}$may exist near the pulsar light cylinder (e.g., Michel 1991). The outer gap model describes the high-requency radiation of the Crab and Vela pulsars fairly well (e.g., Cheng, Ho, \& Ruderman 1986a,b; Romani 1996). Outer gaps may act as a generator of radiation in the pulsar magnetosphere only if the period of the pulsar rotation is small enough, $P<P_{\mathrm{cr}} \simeq$ a few $\times 0.1 \mathrm{~s}$. Optical observations at the positions of $\gamma$-ray pulsars that are near the death line, $P \simeq P_{\mathrm{cr}}$, may test the outer gap model (e.g., Usov 1994; Lundqvist et al. 1999).

\section{Discussion}

For fast rotating pulsars, $P<P_{\mathrm{cr}}$, both polar and outer gaps can act in the pulsar magnetospheres. In this case, the pair plasma properties are very uncertain. For typical pulsars with $P>P_{\mathrm{cr}}$, the polar gap model has no an alternative. If for such pulsars it is confirmed that the distances from the neutron stars to the radio 
emitting regions are in the range $R \ll r_{\text {rad }} \ll c / \Omega$ (Rankin 1993; Kijak \& Gil 1997 and references therein) then most probably, the two-stream instability of the outflowing strongly nonhomogeneous plasma is a reason of the radio emission of pulsars (Usov 1987; Ursov \& Usov 1988; Asseo \& Melikidze 1998). However, if $r_{\text {rad }}$ is either $\sim R$ or $\sim c / \Omega$, the most plausible mechanism of the pulsar radio emission is either the linear acceleration emission at the polar gaps (Melrose 1972) or the cyclotron instability near the light cylinder (Machabeli \& Usov 1979, 1989; Lyutikov et al. 2000).

Acknowledgments. This research was supported by MINERVA Foundation, Munich, Germany.

\section{References}

Arons, J. 1981, ApJ, 248, 1099

Asseo, E., \& Melikidze, G. I. 1998, MNRAS, 301, 59

Cheng, K. S., Ho, C. \& Ruderman, M. A. 1986a,b, ApJ, 300, 500\&522

Kijak, J., \& Gil, J. 1997, MNRAS, 288, 631

Lesch, H., Gil, J. A., \& Shukla, P. K. 1994, Space Sci.Rev., 68, 349

Lundqvist, P., Sollerman, J., Ray, A., Leibundgut, B., \& Sutaria, F. 1999, A\&A, $343, \mathrm{~L} 15$

Lyubarskii, Yu. E. 1996, A\&A, 308, 809

Lyutikov, M., Blandford, R. D., \& Machabeli, G. Z. 2000, this volume

Machabeli, G. Z., \& Usov, V. V. 1979, Soviet Astron. Lett., 5, 238

Machabeli, G. Z., \& Usov, V. V. 1989, Soviet Astron. Lett., 15, 393

Mahajan, S. M., Machabeli, G. Z., \& Rogova, A. D. 1997, ApJ, 478, L129

Melikidze, G. I., Gil, J. A., \& Pataraya, A. D. 2000, this volume

Melrose, D. B. 1978, ApJ, 225, 557

Michel, F. C. 1991, Theory of Neutron Star Magnetospheres, Univ. of Chicago Press

Rankin, J.M. 1993, ApJ, 405, 285

Romani, R. W. 1996, ApJ, 470, 469

Rowe, E. T. 1992, Australian J. Phys., 45, 1

Ruderman, M. A., \& Sutherland, P. G. 1975, ApJ, 196, 51

Shabad, A. E., \& Usov, V. V. 1986, Ap\&SS, 128, 377

Usov, V. V. 1987, ApJ, 320, 333

Usov, V. V. 1994, ApJ, 427, 394

Usov, V. V. 1996, in ASP Conf. Proc., 105, Pulsars : Problems \& Progress, ed.

S. Johnston, M. A. Walker \& M. Bailes (San Francisco: ASP), 323

Usov, V. V., \& Melrose, D. B. 1995, Australian J. Phys., 48, 571

Usov, V. V., \& Melrose, D. B. 1996, ApJ, 464, 306

Ursov, V. N., \& Usov, V. V. 1988, Ap\&SS, 140, 32

Zhang, B., \& Harding, A. K. 1999, astro-ph/9911028 